\title{
The role of heterotrimeric $G$ proteins in the control of symbiosis development in legume plants
}

\author{
Andrey D. Bovin ${ }^{1}$, Irina V. Leppyanen ${ }^{1}$, Olga A. Pavlova ${ }^{1}$, and Elena A. Dolgikh,"* \\ ${ }^{1}$ All-Russia Research Institute for Agricultural Microbiology, Laboratory of signal regulation, \\ 196608 Saint Petersburg, Russia
}

\begin{abstract}
Heterotrimeric G proteins are involved in the regulation of signaling pathways in eukaryotes. Previously, the data about possible participation of heterotrimeric $G$ proteins in the regulation of nodulation in legumes were obtained, however, specific proteins, their composition and role in this process remain poorly understood. In this work searching of the genes encoding the alpha, beta, and gamma subunits of heterotrimeric $\mathrm{G}$ proteins based on an analysis of the Pisum sativum L. genome was performed, as well as the dynamics of the gene expression encoding the particular subunits of $G$ proteins in the process of symbiosis was studied. In addition, a significant effect of beta 1-subunit gene suppression by RNA interference on the nodulation process was revealed.
\end{abstract}

\section{Introduction}

Heterotrimeric G proteins are well-known regulators of signaling pathways in eukaryotes. The most studied are the $G$ proteins in animals and only a few years ago the evidences demonstrating the presence of $G$ proteins in plants were obtained based on the structural similarity of the alpha, beta, and gamma subunits of these proteins [1].

In animals, heterotrimeric $G$ proteins are associated with specific receptors with 7 transmembrane domains (known as G protein-coupled receptors, GPCR) and transmit a signal to other intracellular participants of the signal transduction cascades. In an inactive state the complex consists of alpha, beta, and gamma subunits and is linked to the receptor through the alpha subunit associated with the GDP molecule [2]. When the receptor is activated, the exchange of GDP to GTP occurs on the alpha subunit which leads to the dissociation of the complex. The function of signal transduction is carried out mainly by a complex of beta and gamma subunits. This $\mathrm{G}$ protein activation pattern is mainly common to animals and plants. [3].

However, unlike animals, almost no functional GPCRs were found in plants [4]. The activation mechanisms of heterotrimeric $G$ proteins in plants are more diverse. Plant $G$ proteins either interact with specific RGS-proteins (regulator of G protein signaling, RGS) which have 7 transmembrane domains [3], or directly with various receptors $[5,6]$. In addition, the alpha subunits of plant $\mathrm{G}$ proteins can independently stimulate the exchange of

\footnotetext{
${ }^{*}$ Corresponding author: dol2helen@yahoo.com
} 
guanidine nucleotides, therefore the RGS proteins or receptors in the absence of an external signal inhibit the exchange of GDP for GTP in the associated alpha subunits, thereby supporting the association of the G protein complex (its inactive state)[7].

The interest in studying heterotrimeric plant $G$ proteins as master regulators of signaling pathways is determined by their interaction with a complex of other regulators phospholipases $\mathrm{C}$ and $\mathrm{D}$, protein kinases, ion channels, as well as the effect on ROS formation and calcium metabolism in cells [8-13]. Many of these participants of the signaling pathways are involved in the regulation of the symbiosis development of legume plants with nitrogenfixing rhizobia. [14]. Despite the fact that in the late 1990s the data about the possible participation of heterotrimeric $G$ proteins in the regulation of nodulation were obtained using specific inhibitors and activators [15], the specific G proteins themselves, their composition, and role in signal regulation are still poorly studied.

In the present work, based on the analysis of a recently published pea genome [16] we found genes encoding various subunits of heterotrimeric $\mathrm{G}$ proteins in pea Pisum sativum $\mathrm{L}$. and studied the role of individual subunits in the process of nodulation.

For this purpose, the expression dynamics of the genes encoding various subunits of the $\mathrm{G}$ protein was evaluated during nodulation in wild type pea plants. A genetic construct was obtained to suppress the expression of the Gbetal gene encoding the beta 1-subunit of the G protein using RNA interference and the effect of such suppression on the nodulation of pea $P$. sativum was evaluated.

\section{Materials and methods}

\subsection{Analysis of the gene expression dynamics during nodulation}

4-5 day old seedlings of $P$. sativum cv. Frisson were grown in sterile conditions and transferred into vermiculite wetted with Jensen medium [17], then the plants were inoculated with bacteria of $R$. leguminosarum CIAM1026 (OD600 =0.6). Plant roots $(\mathrm{n}=3)$ were harvested on days $1,3,5,7,9$, and 11 after inoculation. On day 13, 15 and 21, the nodules were harvested. The roots of uninoculated plants collected at similar time points were used as a control. Total RNA was isolated using Trizol reagent (Thermo Fisher, USA). Synthesis of cDNA was performed using reverse transcriptase (Thermo Fisher, USA) on $2.5 \mu \mathrm{g}$ of isolated RNA. Quantitative PCR combined with reverse transcription (RT-PCR) was performed using CFX-96 system (Bio-Rad Laboratories, USA) with SYBR Green dye. The threshold cycle $(\mathrm{Ct})$ values were calculated using the Bio-Rad CFX Manager 1.6 program and analyzed using the 2- $\Delta \Delta \mathrm{Ct}$ method [18]. PCR primers were synthesized by Evrogen (www.evrogen.com). Two biological replicates were analyzed and the results of a representative repeat were shown in the graphs.

\subsection{Obtaining a genetic construct for RNA interference}

To obtain a construct for suppression of the Gbetal gene, fragment of the coding sequence of this gene (227 bp) was amplified with primers containing attB-sites and cloned into the intermediate pDONR221 vector followed by the cloning into the pK7GWIWG2D-IIRedRoot vector for RNA interference.

\subsection{Transformation of pea plants}

$P$. sativum seedlings of cv. Finale were grown under sterile conditions and cut off in the area of the hypocotyl, then a suspension of Agrobacterium rhizogenes Arqual strain carrying the 
necessary construct was applied to the wounded area. 10-14 days after cultivation on solid Jensen medium [17], the seedling were transferred to Emergence medium with cefotaxime and incubated for 3-4 days, the presence of transgenic roots was checked by fluorescence of reporter protein. Plants were transferred to pots with vermiculite, wetted with Jensen solution. 3-4 days after transfer the plants were inoculated with a suspension of bacteria $R$. leguminosarum CIAM 1026 grown up to an optical density of $\mathrm{OD}_{600}=0.5 .21$ days after inoculation the nodule development on the roots of composite plants was assessed.

\section{Results and discussion}

\subsection{Search for genes encoding various subunits of heterotrimeric $\mathbf{G}$ proteins in the pea genome}

To assess the diversity of heterotrimeric $\mathrm{G}$ proteins in $P$. sativum, a genome analysis was performed [16] for the presence of genes encoding the alpha, beta, and gamma subunits of these regulators. Screening was performed using the known coding sequences of the alpha, beta, and gamma subunits of two model legume plants Medicago truncatula and Glycine max [19], for which these sequences were previously annotated in the NCBI and Phytozome databases. Identified pea sequences and their homologs in M. truncatula and G. max are presented in table 1 .

Table 1. The list of heterotrimeric G proteins subunits genes of $P$. sativum

\begin{tabular}{|c|c|c|c|c|}
\hline \multirow[b]{2}{*}{ Subunit } & \multirow[b]{2}{*}{ Gene } & \multicolumn{3}{|c|}{ Accession number } \\
\hline & & $\begin{array}{c}\text { Pisum sativum v1a } \\
{[16]}\end{array}$ & $\begin{array}{c}\text { Medicago } \\
\text { truncatula Mt4.0v1 }\end{array}$ & $\begin{array}{l}\text { Glycine max } \\
\text { Wm82.a2.v1 }\end{array}$ \\
\hline Alpha 1 & Galphal & Psat6g021680 & Medtr1g015750.1 & $\begin{array}{l}\text { Glyma.17G226700 } \\
\text { Glyma.14G098000 }\end{array}$ \\
\hline Alpha 2 & Galpha2 & Psat5g034360 & Medtr3g105240 & $\begin{array}{l}\text { Glyma.04G056600 } \\
\text { Glyma.06G057000 }\end{array}$ \\
\hline Alpha 3 & Galpha3 & Psat7g167760 & Medtr3g105240 & $\begin{array}{l}\text { Glyma.04G056600 } \\
\text { Glyma.06G057000 }\end{array}$ \\
\hline Beta 1 & Gbetal & Psat5g006200 & Medtr3g116500 & $\begin{array}{l}\text { Glyma.06G013000 } \\
\text { Glyma.12G043900 } \\
\text { Glyma.04G013100 } \\
\text { Glyma.11G118500 }\end{array}$ \\
\hline Beta 2 & Gbeta2 & Psat7g003840 & Medtr8g106790 & Glyma.05G243800 \\
\hline Beta 3 & Gbeta3 & Psat5g014800 & Medtr3g113190 & Glyma.06G026400 \\
\hline Gamma 1 & Ggammal & Psat0s2465g0080 & Medtr1g071100 & $\begin{array}{l}\text { Glyma.10G030900 } \\
\text { Glyma.02G143500 }\end{array}$ \\
\hline Gamma 2 & Ggamma2 & Psat6g114080.1 & $\begin{array}{l}\text { Medtr1g076580, } \\
\text { Medtr1g076640 }\end{array}$ & $\begin{array}{l}\text { Glyma.20G192300 } \\
\text { Glyma.10G178700 }\end{array}$ \\
\hline Gamma 3 & Ggamma3 & Psat6g031800 & Medtr1g010050 & $\begin{array}{c}\text { Glyma.U010900, } \\
\text { Glyma.14G132400 } \\
\text { Glyma.17G200900 }\end{array}$ \\
\hline Gamma 4 & Ggamma4 & Psat1g134600 & Medtr2g042200 & $\begin{array}{l}\text { Glyma.15G178100 } \\
\text { Glyma.17G048600 }\end{array}$ \\
\hline Gamma 5 & Ggamma5 & Psat4g168440 & Medtr8g021170 & Glyma.07G040200 \\
\hline Gamma 6 & Ggamma6 & Psat4g018440 & Medtr4g125015 & Glyma.13G110900 \\
\hline
\end{tabular}


Finally, genes encoding 3 alpha subunits, 3 beta subunits, and 6 gamma subunits were identified in the genome of pea.

\subsection{Expression of the genes encoding various subunits of heterotrimeric $\mathbf{G}$ proteins in the roots of wild type pea plants of the cv. Frisson during nodulation}

To assess the role of heterotrimeric $\mathrm{G}$ proteins in the process of nodule formation we analyzed the expression dynamics of the genes encoding various subunits of pea heterotrimeric $G$ proteins (Fig. 1). For analysis wild type pea plants (cv. Frisson) harvested at different time points after inoculation were used.
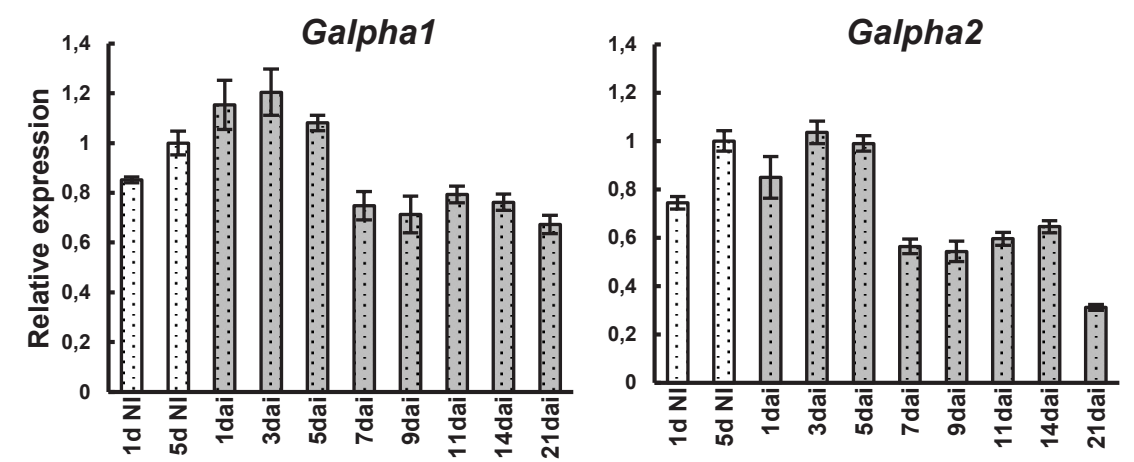

Fig. 1 Expression dynamics of the Galpha1 and Galpha2 genes encoding the alpha 1- and alpha 2subunits of the $\mathrm{G}$ protein in pea roots and nodules at $1,3,5,7,9,11,13,15$, and 21 days after inoculation in relation to uninoculated variants ( $1 \mathrm{~d} \mathrm{NI}$ and $5 \mathrm{~d} \mathrm{NI})$. Bars represent $\pm \mathrm{SEM}$. Two biological replicates were analyzed and the results of a representative repeat were shown in the graphs.

In wild type pea plants the expression of the Galpha1 and Galpha2 genes did not change significantly at the early stages after inoculation while at later points a slight decrease in the expression level of these two genes was found.
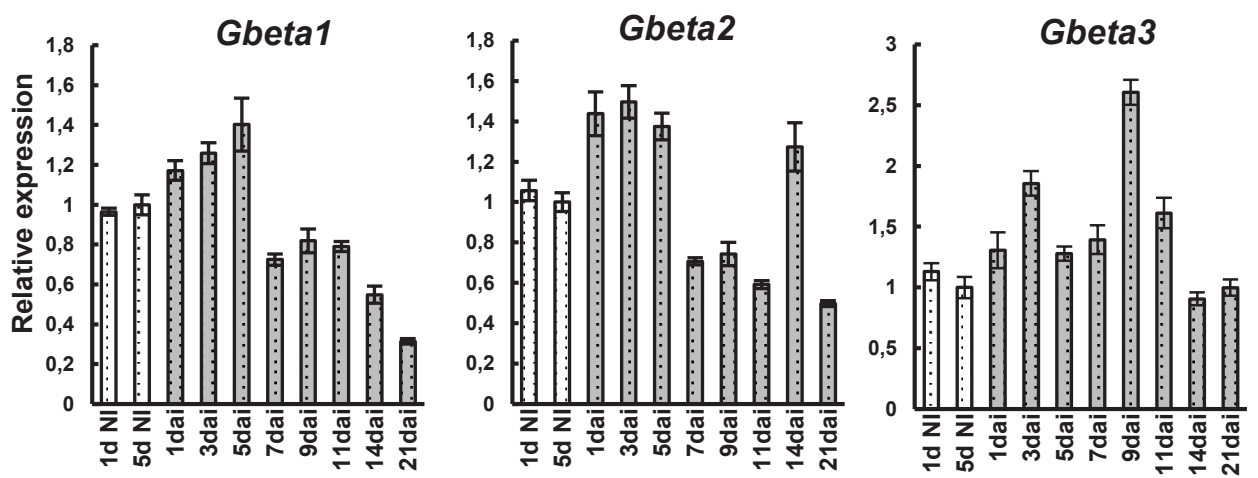

Fig. 2 Expression dynamics of the Gbeta1, Gbeta 2 и Gbeta 3 genes encoding the beta 1, 2 и 3subunits of the $\mathrm{G}$ protein in pea roots and nodules at $1,3,5,7,9,11,13,15$, and 21 days after inoculation in relation to uninoculated variants (1d NI and 5d NI). Bars represent SEM. Two biological replicates were analyzed and the results of a representative repeat were shown in the graphs. 
In contrast, analysis of the expression of the Gbeta1, Gbeta2, and Gbeta3 genes encoding the beta 1-, beta 2-, and beta 3-subunits of the G protein (Fig. 2) showed a small but significant increase at 1-3 days after inoculation. In pea nodules the expression of the Gbeta 3 gene was increased. This may indicate the involvement of beta $1-$, beta 2 - and beta 3 -subunits of the $\mathrm{G}$ protein in the control of the early stages of the symbiosis development in pea.

Thus, analysis of the expression dynamics of the genes encoding various subunits of heterotrimeric $\mathrm{G}$ proteins showed that at the early stages of symbiosis the Gbetal, Gbeta 2 and Gbeta 3 pea genes can be activated. Since the downstream signal transduction mainly requires the beta and gamma subunits, at the next stage of our research the role of individual beta subunits in controlling the symbiosis development was studied in details.

\subsection{Effect of suppression of Gbeta1 gene encoding the $P$. sativum beta 1- subunit of the heterotrimeric $\mathbf{G}$ protein on the nodulation process}

To assess the effect of the beta 1-subunit of the heterotrimeric $G$ protein in symbiosis development, the construct for suppression of the Gbetal gene by RNA interference was obtained. As a result of this gene suppression, nodule formation on the roots of Gbetal-RNAi plants was significantly reduced compared to control plants transformed with the $G U S$ construct in two independent experiments (a 6-fold decrease $(\mathrm{p}=0,0004)$ ) (fig. 3). Based on these data, it can be concluded that the beta 1-subunit is necessary for the positive regulation of nodulation.

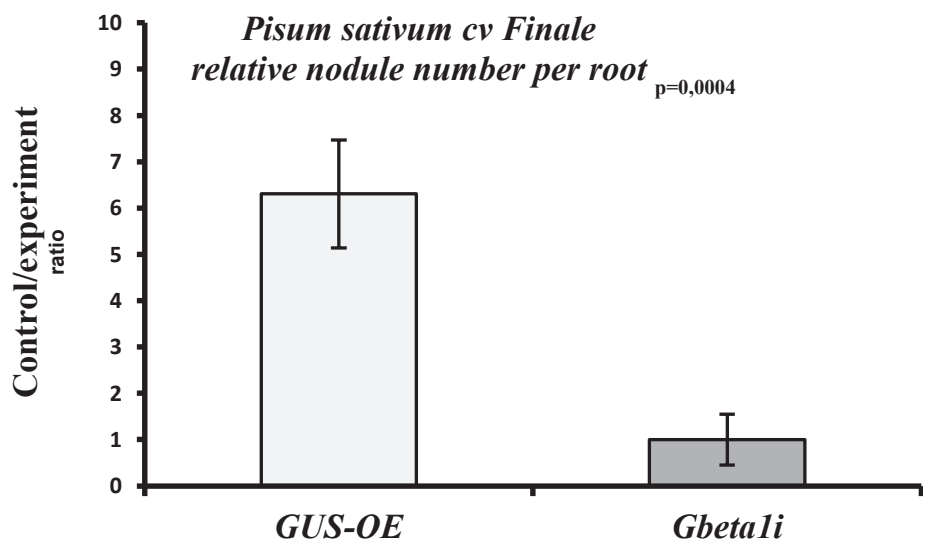

Fig 3. The effect of suppression of the Gbetal gene encoding the beta 1-subunit of the G protein on nodulation in pea plants. GUS-OE - control plants transformed with the GUS construct. Bars represent \pm SEM. The level of significance was presented.

\section{Conclusion}

As a result, we were able to identify 3 alpha, 3 beta, and 6 gamma subunits of a heterotrimeric $\mathrm{G}$ protein in peas. Analysis of the dynamics of gene expression showed that beta 1-, beta 2-, and beta 3-subunits of G-protein can be involved in the control of the early stages of symbiosis. Indeed, suppression of the Gbetal gene encoding the beta 1 subunit led to a significant decrease in the number of nodules formed on the roots of pea plants. Further studies should reveal the receptors and components of signal transduction pathway that interact with the beta 1-subunit of $G$ protein.

The research was supported by RSF (project No. 16-16-10043). 


\section{References}

1. H. Ullah, J.-G. Chen, B. Temple, D. C. Boyes, J. M. Alonso, K. R. Davis, J. R. Ecker, and A. M. Jones, Plant Cell 15, 393 (2003). DOI: 10.1105/tpc.006148

2. E. J. Neer, Protein Sci. 3, 3 (1994). DOI: 10.1002/pro.5560030102

3. D. Stateczny, J. Oppenheimer, P. Bommert, Curr. Opin. Plant Biol. 34, 127 (2016). DOI: 10.1016/j.pbi.2016.11.001

4. Y. Trusov, J. R. Botella, Front. Chem. 4, 1 (2016). DOI: 10.3389/fchem.2016.00024

5. X. Liang, P. Ding, K. Lian, J. Wang, M. Ma, L. Li, L. Li, M. Li, X. Zhang, S. Chen, Y. Zhang, J.-M. Zhou, Elife 5 (2016). DOI: 10.7554/eLife. 13568

6. M. N. Aranda-Sicilia, Y. Trusov, N. Maruta, D. Chakravorty, Y. Zhang, J. R. Botella, J. Plant Physiol. 188, 44 (2015). DOI: 10.1016/j.jplph.2015.09.005

7. D. Urano, J. C. Jones, H. Wang, M. Matthews, W. Bradford, J. L. Bennetzen, A. M. Jones, PLoS Genet. 8 (2012). DOI: 10.1371/journal.pgen.1002756

8. J. Sun, X. Liu, Y. Pan, Front. Agric. China 5, 64 (2011)

9. S. R. Choudhury, S. Pandey, Plant J. 86, 50 (2016). DOI: 10.1111/tpj.13151

10. M. den Hartog, A. Musgrave, T. Munnik, Plant J. 25, 55 (2001). DOI: 10.1046/j.1365313x.2001.00931.x

11. A. Nakashima, L. Chen, N. P. Thao, M. Fujiwara, H. L. Wong, M. Kuwano, K. Umemura, K. Shirasu, T. Kawasaki, K. Shimamoto, Plant Cell. 20, 2265 (2008). DOI: $10.1105 /$ tpc. 107.054395

12. X.-Q. Wang, H. Ullah, A. M. Jones, S. M. Assmann, Science 292, 2070 (2001). DOI: 10.1126/science. 1059046

13. L.-M. Fan, W. Zhang, J.-G. Chen, J. P. Taylor, A. M. Jones, S. M. Assmann, Proc. Natl. Acad. Sci. USA 105, 8476 (2008). DOI: 10.1073/pnas.0800980105

14. C. Zipfel, G. E. D. Oldroyd, Nature 543, 328 (2017). DOI: 10.1038/nature22009

15. J.-L. Pingret, E. P. Journet, D. G. Barker, Plant Cell. 10, 659 (1998). DOI: 10.1105/tpc.10.5.659

16. J. Kreplak, M.-A. Madoui, P. Cápal, P. Novák, K. Labadie, G. Aubert, P. E. Bayer, K. K. Gali, R. A. Syme, D. Main, A. Klein, A. Bérard, I. Vrbová, C. Fournier, L. d'Agata, C. Belser, W. Berrabah, H. Toegelová, Z. Milec, J. Vrána, H. Lee, A. Kougbeadjo, M. Térézol, C. Huneau, C. J. Turo, N. Mohellibi, P. Neumann, M. Falque, K. Gallardo, R. McGee, B. Tar'an, A. Bendahmane, J.-M. Aury, J. Batley, M.-C. Le Paslier, N. Ellis, T. D. Warkentin, C. J. Coyne, J. Salse, D. Edwards, J. Lichtenzveig, J. Macas, J. Doležel, P. Wincker, J. Burstin, Nat. Genet. 51, 1411 (2019). DOI: 10.1038/s41588-019-0480-1

17. A. A. van Brussel, T. Tak, A. Wetselaar, E. Pees, C. Wijffelman, Plant Sci. Lett. 27, 317 (1982)

18. K. J. Livak, T. D. Schmittgen, Methods 25, 402 (2001). DOI: 10.1006/meth.2001.1262

19. S. R. Choudhury, S. Pandey, Plant Physiol. 162, 522 (2013). DOI: 10.1104/pp.113.215400 